\title{
Managing Manufacturing Risks by Using Capacity Options ${ }^{\mathrm{I}}$
}

\author{
BARIŞ TAN \\ Graduate School of Business, Koç University \\ Rumeli Feneri Yolu, Sarıer, 80910 Istanbul, Turkey \\ e-mail:btan@ku.edu.tr
}

\begin{abstract}
August 200I
Abstract

In this study, we investigate the strategy of increasing production capacity temporarily through contingent contractual agreements with short-cycle manufacturers to manage the risks associated with demand volatility. We view all these agreements as capacity options. More specifically, we consider a manufacturing company that produces a replenishment product that is sold at a retailer. The demand for the product switches randomly between a high level and a low level. The production system has enough capacity to meet the demand in the long run. However, when the demand is high, it does not have enough capacity to meet the instantaneous demand and thus has to produce to stock in advance. Alternatively, a contractual agreement with a short-cycle manufacturer can be made. This option gives the right to receive additional production capacity when needed. There is a fixed cost to purchase this option for a period of time and if the option is exercised, there is an additional per unit exercise price which corresponds to the cost of the goods produced at the short-cycle manufacturer. We formulate the problem as a stochastic optimal control problem and analyze it analytically. By comparing the costs between two cases where the contract with the shortcycle manufacturer is used or not, the value of this option is evaluated. Furthermore, the effect of demand variability on this contract is investigated.
\end{abstract}

Key Words: Options, Capacity planning, Stochastic Modeling

\footnotetext{
I Pre-print : Tan, B, "Managing Manufacturing Risks by Using Capacity Options," Journal of the Operational Research Society, Vol. 53, No. 2, pp. 232-242, February 2002
} 


\title{
Managing Manufacturing Risks by Using Capacity Options
}

\author{
BARIŞ TAN
}

\section{Introduction}

\section{Motivation}

This study is motivated by the challenges in the retail-apparel-textile channel. The retail-appareltextile channel is characterized by rapidly changing styles, volatile customer demand, product proliferation, and long lead times.

The risks that both retailers and manufacturers face increase as the demand volatility increases. Facing with increased product proliferation and demand volatility, retailers are adopting lean retailing practices to manage the risks associated with having too much or too little inventory'.

One of the strategies to reduce these risks is to delay production and ordering decisions to incorporate revised demand forecasts ${ }^{2,3}$. Consequently, lean retailers demand fulfillment of a higher percentage of their orders within a selling season from their suppliers. This change in the retailing industry has increased the demand volatility risks a manufacturer faces. In order to cope with these challenges, a manufacturer should devise effective strategies to respond effectively to changes in demand.

From a manufacturer's perspective, the ways to respond quickly to changes in demand are producing to stock in advance, increasing the production capacity permanently by investing in new production facilities, increasing the production capacity temporarily by using overtime, subcontracting, etc., and combinations of these pure strategies. Making these decisions in the most effective way is vital for a manufacturer to be competitive.

Traditionally, sourcing decisions are based on product costingmethods that often do not take above-mentioned risks into account. As a result, manufacturing companies who shifted their production to overseas suppliers with long lead times in quest of lower production costs rely on high inventories to respond quickly to changes in customer demand. However, using a supplier with a short lead-time but higher production cost can be more profitable, especially, for goods with high demand variability. Simulation studies show that additional costs of using a manufacturer with a short lead time can be justified by reducing the inventory levels and its associated costs ${ }^{4}$.

\section{Goal of the Paper}

The goal of this study is to investigate the strategy of increasing the production capacity temporarily through contingent contractual agreements with short-cycle manufacturers to manage the risks associated with demand uncertainty analytically. 
In order to evaluate this strategy, we view the agreements with a short-cycle manufacturer as real options. An option is the right, but not obligation, to take an action in the future and a real option is the extension of financial option theory on real (non-financial) assets ${ }^{5}$. Since the contractual agreement is related to increasing the capacity, we refer this option as a capacity option.

More specifically, we consider a manufacturing company that produces a replenishment product that is sold at a retailer. The demand for the product switches randomly between a high level and a low level. The production system has enough capacity to meet the demand in the long run. However, when the demand is high, it does not have enough capacity to meet the demand by production and has to produce to stock in advance. Alternatively, a contractual agreement with a short-cycle manufacturer can be made. The short-cycle manufacturer can deliver the requested goods in a very short time which can be negligible compared to the other sourcing alternatives. This option gives the right to receive additional production capacity when needed. There is a fixed cost to purchase this option for a period of time and if the option is exercised, there is an additional per unit exercise cost which corresponds to the cost of the goods produced at the shortcycle manufacturer.

After framing the strategy in the options terminology, the question we ask in order to evaluate this strategy is: What is the value of a given capacity option? The value of the option depends on the terms of the contract including option price, the exercise cost, its duration, our capacity, and the demand volatility. In order to answer this question, we need to solve the following problem: How do we operate our manufacturing plant, and how do we use a subcontractor to maximize profits?

\section{Approach}

We form a stochastic dynamic programming problem which is an extension of a widely studied stochastic production control problem where a factory manager had to decide how to operate an unreliable machine to satisfy a constant demand ${ }^{6}$.

The problem considered in this study differs from this basic model in three ways: First, both the manufacturing facility and the subcontractor are perfectly reliable and randomness in the model comes from the variability of demand. A two-state alternating renewal process with exponential switching times is used to model customer arrivals. That is, the demand rate is either high or low, and the time to switch from high to low and low to high are exponentially distributed random variables. Second, it is possible to increase the production capacity temporarily. In this way, if demand temporarily exceeds capacity, the manager may purchase some of the product from others to reduce backlog and improve service to customers. However, the profit made from purchased finished goods is less than that from items produced in-house. Finally, the objective function is profit maximization rather than cost minimization. Profit is the money generated through sales by production at the manufacturing facility and at the subcontractor minus the inventory and backlog costs. 
The Bellman equation is used to determine the structure of the problem. It is found that the structure is the same as the one given for the unreliable production and constant demand case ${ }^{7}$. The optimal policy for the manufacturing facility is to produce with the maximum rate until a desired finished good inventory level is reached and produce with the demand rate at this level. The subcontractor is only used when the backlog level reaches a certain level and it is used at a rate to keep the backlog at this level.

In order to determine the optimal values of these thresholds, first the differential equations for the density functions of the surplus/backlog are derived and solved analytically. Then the objective function is expressed as a function of the thresholds. Finally, the optimal values of the thresholds are determined by optimizing the objective function over the thresholds.

Organization of the remaining part of this paper is as follows: In the next section, a review of the pertinent literature is given. Then the basic model and its assumptions are given. Next, the production control problem is formulated and the optimal policy is characterized. Following this characterization, the model using the optimal policy is analyzed. Then, an analysis of the terms of a given contract by using the methodology is presented. Finally, the concluding remarks are given.

\section{Past Work}

In this section, we review the pertinent literature on contractual agreements with suppliers and subcontracting policies.

\section{Capacity Options}

Jain and Silver ${ }^{8}$ investigate a case where both the demand and the capacity of the supplier are uncertain and the capacity of the supplier can be reserved by paying a premium. The replenishment quantity and the amount of capacity to reserve are determined to minimize the overall costs. Costa and Silver ${ }^{9}$ extend this approach to a multiperiod analysis.

In the apparel catalog industry, contracts similar to options are used. Eppen and Iyer ${ }^{\text {10 }}$ present backup agreements used in apparel catalog industry. Under this agreement, the buyer makes a firm commitment to purchase a given number of goods at the beginning of the horizon. In the first period, the buyer purchases a certain percentage of this commitment, $\rho$, at a given price. At the second period, if the buyer purchases less than the committed, a penalty of $b$ dollars per unit is paid for the remaining parts that are not purchased. It is reported that a catalog company Catco uses these contracts with Anne Klein and DKNY $(\rho=0.2, b=0)$ and with Liz Claiborne $(\rho=0.25, b=0.2 c)^{\text {IO }}$.

Another contract type is the quantity flexibility contract ${ }^{\mathrm{II}}$. Under a quantity flexibility contract, the buyer provides a forecast of future orders to the supplier. Later, the buyer purchases between a predetermined minimum and the maximum levels within the initial forecasts. That is, a minimum quantity needs to be purchased at the agreed price and there is an option to purchase up to the maximum level at the same price. The buyer can update the forecasts later on. These 
contracts are reported to be used in the electronics industry ${ }^{12,13}$, for example, by the IBM printer division, Sun Microsystems, Solectron, Hewlett Packard, etc.

If the capacity is scarce, a buyer may pay an upfront fee to reserve capacity in advance. For example, in the semiconductor industry, according to a recent survey by the Fabless Semiconductor Association, $30 \%$ of capacity reservation arrangements are take-or-pay. However, agreements called pay-to-delay capacity reservation are also used ${ }^{14}$. Under this agreement, the buyer makes an agreement with the supplier to purchase a minimum quantity at a given price $c_{\mathrm{f}}$ and pays $c_{o}$ per unit to reserve up to a level. These additional units can be purchased at an extra unit cost of $c_{e}$.

In a recent study, the role of options is examined in a two-stage buyer-supplier system ${ }^{15}$. Using a two-period model with correlated demand, it is shown that options provide flexibility to a buyer to respond to market changes in the second period. They also investigate the implications of such arrangements for coordination of the supply chain. They argue that return policies could be used that coordinate the channel and give the supplier positive profits. In this study, all three agreements discussed above are discussed as special cases of a general option.

Note that most of these studies consider two period models and therefore cannot be used directly to analyze the policies for replenishment products where subcontracting can be used at any given time.

\section{Subcontracting}

Modeling the problem as a stochastic optimal control problem and determining the feedback policy allows us to analyze the strategy of increasing the production capacity temporarily for replenishment products.

Gershwin discusses an extension of a Flexible Manufacturing System (FMS) scheduling model where the capacity of the FMS can be increased, if necessary ${ }^{16}$. For a long-term increase, more machines can be bought or more operators can be hired, while for a short-term increase, excess demand can be contracted or labor hours can be increased with overtime. He shows that the optimal policy is similar to an $(\mathrm{s}, \mathrm{S})$ policy where subcontracting is used when the inventory position goes down to a lower hedging level and the parts are produced with the maximum rate until an upper hedging level is reached. The optimality of the hedging point policy for this problem is also proven ${ }^{7}$. Both of these models assume that the demand is constant and the production rate of the machine and also the rate at which the contractor can supply goods are greater than the demand rate. Furthermore, the objective is to minimize the average inventory carrying, backlog costs and cost of increased capacity.

For a number of discrete-time inventory models with two sources, a dual base-stock policy, with parameters $s_{1}$ and $s_{2}$, is shown to be optimal ${ }^{17}$, ${ }^{8}$. In this policy, the first source, the manufacturer, is used when the inventory falls below $s_{\mathrm{I}}$ and the second source, the subcontractor, is used when the inventory falls below $s_{2}$. 
Similarly, in a model where backlogging is not allowed and a subcontractor can be used by paying a fixed cost and a variable cost, a Brownian approximation of the model is used to show that a double band policy is optimal for the problem of controlling a machine that can produce at a fixed rate to meet random demand ${ }^{19}$.

A Brownian motion approximation for the optimal subcontracting policy for an $\mathrm{M} / \mathrm{M} / \mathrm{I}$ system is also shown to a two threshold policy ${ }^{20}$. This policy is utilized to analyze capacity, inventory policies and also competition and coordination issues ${ }^{21}$.

In this study, we do not consider competition among manufacturers and subcontractors. In a two-period model, a competitive stochastic investment game is analyzed ${ }^{22}$. In this model, the manufacturer and subcontractor decide on their capacity investment levels separately in the first period. After the demand uncertainty is resolved in the second period, both parties decide on their production and sales with the option to subcontract. The value of the option of subcontracting is then determined by using this model.

In this study, both production and subcontracting policies are determined directly by formulating the problem as a stochastic dynamic programming problem with continuous flow. This approach allows us to handle demand variability, production and subcontracting capacity limits to value capacity options for manufacturers that produce a replenishment product.

\section{Model Description}

We consider a manufacturing company that produces a replenishment product that is sold at a retailer. The production rate of the manufacturing facility at time $t$, denoted by $u(t)$ is one of the decision variables. The manufacturing company can increase its capacity temporarily by receiving additional production from a subcontractor. The rate at which the subcontractor is asked to deliver goods at time $t$, denoted by $v(t)$, is the other decision variable. The demand rate from the retailer to the manufacturing company at time $t$ is denoted by $d(t)$. The difference between the cumulative production and cumulative demand at time $t$ is denoted by $x(t)$ which is the finished goods inventory level when $x(t) \geq 0$ and the backlog level when $x(t)<0$. Figure I shows the retailer-manufacturer and subcontractor model considered in this study. 


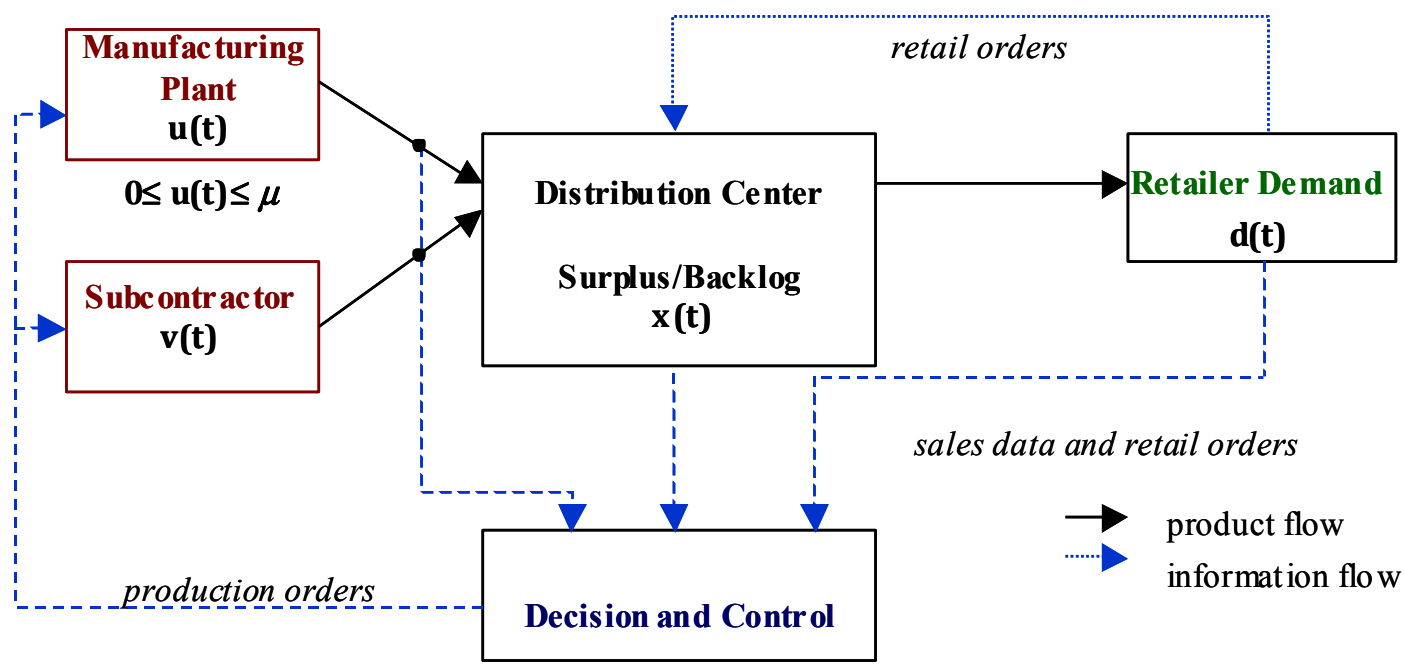

Figure I. Retailer, Manufacturer and Subcontractor product and information flows

\section{Demand}

A two-state alternating renewal process is utilized to represent demand volatility. The state of the demand at time $t$ is $D(t)$ which is either high $(\mathrm{H})$ or low $(\mathrm{L})$. When the demand is high, the demand rate is $d(t)=\mu_{\mathrm{H}}$ and when the demand is low, the demand rate is $d(t)=\mu_{\mathrm{L}}$. The times to switch from a high demand state to a low demand state and from a low demand state to a high demand state are assumed to be exponentially distributed random variables with rates $p$ and $r$. This representation is suitable for describing demand which is stationary in the long run, but whose mean shifts temporarily as a result of unpredictable effects. The mean and variance of the amount of demand that will arrive per unit time are given $\mathrm{as}^{23}$ :

$$
\begin{aligned}
& \bar{d}=\lim _{t \rightarrow \infty} \frac{E[N(t)]}{t}=\mu_{\mathrm{L}}+\left(\mu_{\mathbf{H}}-\mu_{\mathrm{L}}\right) e \\
& \sigma^{2}=\lim _{\mathrm{t} \rightarrow \infty} \frac{\operatorname{Var}[\mathrm{N}(\mathrm{t})]}{\mathrm{t}}=2\left(\mu_{\mathrm{H}}-\mu_{\mathrm{L}}\right)^{2} \frac{1}{\mathrm{r}}(1-\mathrm{e}) \mathrm{e}^{2}
\end{aligned}
$$

where $e=\frac{r}{p+r}$ is the percentage of the time the demand is high and $\mathrm{N}(\mathrm{t})$ is the total demand that arrives during $[0, t)$. The asymptotic distribution of $\mathrm{N}(\mathrm{t})$ is normal ${ }^{23}$. The coefficient of variation of demand is defined as $\mathrm{cv}=\sigma / \overline{\mathrm{d}}$ and used as a summary measure for demand volatility. Figure 2 depicts a simulation of total demand that arrives in to time unit periods that is generated by the alternating demand model considered in this study. 


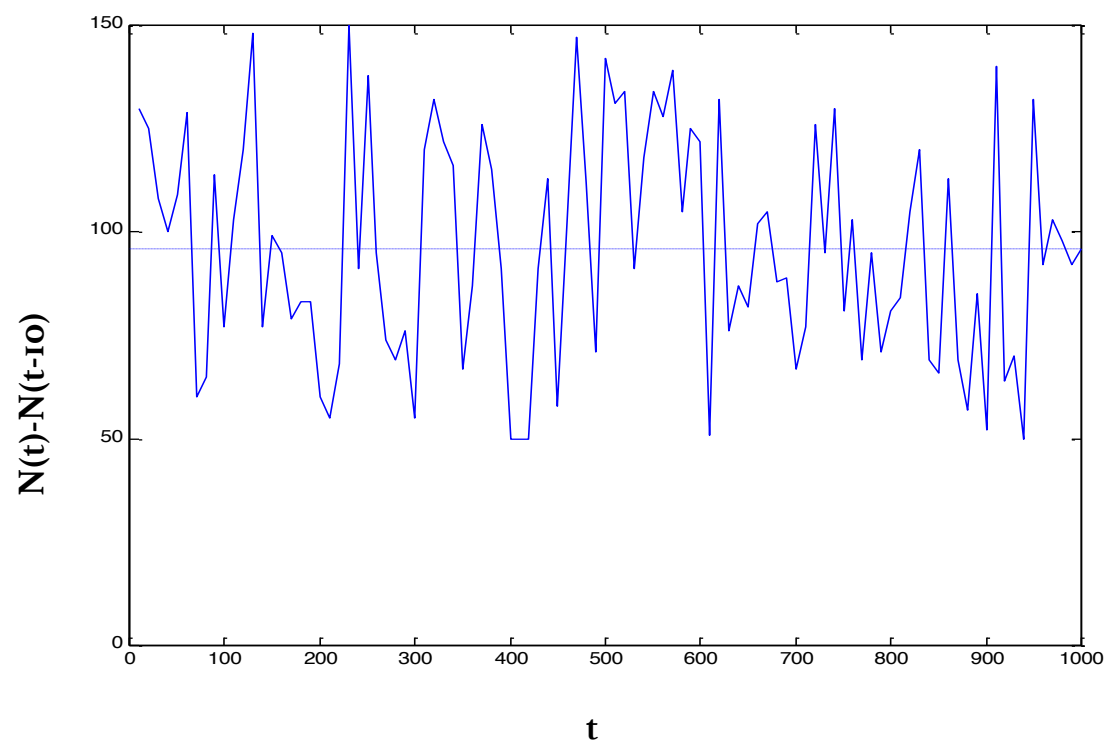

Figure 2. Simulated demand sequence generated by the alternating demand model.

(Accumulated demand in to time unit intervals, $\mu_{\mathrm{H}}=\mathbf{I} .5, \mu_{\mathrm{L}}=\mathbf{0 . 5}, \overline{\mathrm{d}}=0.9, \mathrm{cv}=\mathrm{I}$ )

\section{Manufacturer}

The maximum production rate of the manufacturing facility is $\mu, 0 \leq u(t) \leq \mu$. We assume that the production capacity is sufficient to meet the demand when it is low but insufficient when it is high, i.e., $\mu_{\mathrm{L}}<\mu<\mu_{\mathrm{H}}$. However, it has enough capacity to meet the demand in the long run, i.e., $\mu>\bar{d}$. Note that, if $\mu \geq \mu_{\mathrm{H}}$, the problem is trivial: it is possible to keep zero inventory and a chase strategy that sets the production rate equal to the demand rate is optimal and no subcontracting is used. If $\mu<\mu_{\mathrm{L}}$, subcontracting must be used all the time. It is also assumed that there is no production delay and the manufacturing facility is perfectly reliable.

The profit generated through the sales of the goods produced at the manufacturing facility is $\mathrm{L}$ (dollars per unit). The inventory carrying cost is $\mathrm{c}^{+}$and the backlog cost is $\mathrm{c}^{-}$.

\section{Subcontractor and the Capacity Option}

The maximum production rate of the manufacturing facility is $\mu_{\mathrm{c}}, \mathbf{o} \leq \mathbf{v}(\mathrm{t}) \leq \mu_{\mathrm{c}}$. It is possible to meet the high demand with the additional production received from the subcontractor, i.e., $\mu+\mu_{c} \geq$ $\mu_{\mathrm{H}}$. The subcontractor is also perfectly reliable and they can deliver the requested goods without a delay, hence referred as a short-cycle manufacturer.

The agreement with the subcontractor is considered as a capacity option. The company pays an upfront fee of $\mathrm{C}_{\mathrm{O}}$ to have the right to access additional capacity of $\mu_{\mathrm{c}}$ when it is needed during the duration of the option $\Gamma$. At time $t$, if the company decides to receive additional capacity from the subcontractor, the option is exercised at a price of $\Delta_{c}$ (dollars per unit). The exercise price of the option corresponds to the production cost when it is obtained from the subcontractor. After 
paying the additional cost, the profit generated through the sales of the goods received from the subcontractor is $\mathrm{A}=\mathrm{L}-\Delta_{\mathrm{c}}$ (dollars per unit).

\section{Production and Subcontracting Policy}

\section{Production Control Problem}

The production control problem is modeled as a stochastic optimal control problem by modeling the product flow as a continuous flow. The decision variables of the production control problem are the rate at which the goods are produced at the manufacturing facility at time $t, u(t)$, and the rate at which the subcontractor is requested to supply the goods at time $t, v(t)$. The profit function to be maximized is the difference between the money generated through sales and the inventory carrying and backlog costs. A linear inventory carrying and backlog cost function is assumed:

$$
g(x)= \begin{cases}c^{+} x & x \geq 0 \\ c^{-} x & x<0\end{cases}
$$

Then the production control problem is

$$
V=\max _{u, v} \Pi=E\left[\int_{0}^{T} L u(t)+A v(t)-g(x(t)) d t\right]
$$

subject to

$$
\begin{aligned}
& \frac{d}{d t} x(t)=u(t)+v(t)-d(t) \\
& 0 \leq u(t) \leq \mu \\
& 0 \leq v(t) \leq \mu_{c} \\
& d(t)=\left\{\begin{array}{cc}
\mu_{\mathbf{H}} & \text { if } D(t)=\mathbf{H} \\
\mu_{\mathbf{L}} & \text { if } D(t)=\mathbf{L}
\end{array}\right.
\end{aligned}
$$

Markov dynamics for $\mathrm{D}(\mathrm{t})$ with rates $\mathrm{p}$ (from $\mathrm{H}$ to $\mathrm{L}$ ) and $\mathrm{r}$ (from $\mathrm{D}$ to $\mathrm{U})$

Equation (4) shows the dynamics of $x(t)$. Namely, the rate at which the surplus/backlog changes is equal to the difference between the total supply from the manufacturing facility and the subcontractor and the demand rate. Equations (5) and (6) give the constraints on the decision variables. Finally, equation (7) depicts the randomness in the model that comes from the variability of the demand.

We assume that $T$ is very large, so the optimal policy that is discussed in the next section is based on the assumption that the probability distribution of $(x(t), D(t))$ reaches a steady state. 


\section{Characterization of the Policy}

The production control policy for an unreliable station with a constant demand source and a subcontractor is derived ${ }^{16}$ and rigorously proven for an average cost criterion ${ }^{7}$. In this section, this methodology is followed to state the optimal policy for the variable demand and profit maximization case.

Problem (2)-(7) is a dynamic programming problem. Therefore the solution of the problem, $u(t)$ and $v(t)$ as a function of $x, D, t$ satisfies the Bellman equation ${ }^{24}$. The Bellman equation is used to determine the structure of the optimal policy. The value function is defined as

$$
V(x(t), D(t), t)=\min _{u, v} E\left[\int_{t}^{T}-L u-A v+g(x) d \tau\right]
$$

V satisfies the maximum principle which states, for $D=L$,

$$
-\frac{\partial V}{\partial t}(x, \mathbf{L}, t)=\min _{u, v}\left\{-L u-A v+g(x)+\frac{\partial V}{\partial x}(x, \mathbf{L}, t)\left(u+v-\mu_{\mathbf{L}}\right)+V(x, \mathbf{H}, t) p-V(x, \mathbf{L}, t) p\right\}
$$

and for $\mathrm{D}=\mathrm{H}$,

$$
-\frac{\partial V}{\partial t}(x, \mathbf{H}, t)=\min _{u, v}\left\{-L u-A v+g(x)+\frac{\partial V}{\partial x}(x, \mathbf{H}, t)\left(u+v-\mu_{\mathbf{H}}\right)+V(x, \mathbf{L}, t) r-V(x, \mathbf{H}, t) r\right\}
$$

Equation (8) and (9) yield the optimal feedback policy for $u(t)$ and $v(t)$ in terms of $x(t)$ and the demand state:

$$
\begin{aligned}
& u^{*}(x, D)=\left\{\begin{array}{cl}
0 & \text { if } \quad Z_{u}(D)=-L+\frac{\partial V}{\partial x}(x, D)>0 \\
\text { undetermined } & \text { if } \quad Z_{u}(D)=-L+\frac{\partial V}{\partial x}(x, D)=0, \mathrm{D}=\mathbf{H}, \mathbf{L} \\
\mu & \text { if } \quad Z_{u}(D)=-L+\frac{\partial V}{\partial x}(x, D)<0
\end{array}\right. \\
& v^{*}(x, D)=\left\{\begin{array}{cl}
\text { if } \quad Z_{v}(D)=-A+\frac{\partial V}{\partial x}(x, D)>0 \\
0 & \text { if } Z_{v}(D)=-A+\frac{\partial V}{\partial x}(x, D)=0, \mathrm{D}=\mathbf{H}, \mathbf{L} \\
\mu & \text { if } \quad Z_{v}(D)=-A+\frac{\partial V}{\partial x}(x, D)<0
\end{array}\right.
\end{aligned}
$$


Since $\mathrm{V}$ is a solution of a dynamic programming problem, it is plausible to assume that $\mathrm{V}$ is strictly convex in $\mathrm{x}$ with a unique minimum. Therefore, for the manufacturer there is a value of $\mathrm{x}$, $Z_{u}(D)$, for each demand state, which $u=\mu$ if $x<Z_{u}(D)$ and $u=0$ if $x>Z_{u}(D)$. Similarly, for the subcontractor, there is a value of $x, Z_{v}(D)$, which $v=\mu_{c}$ if $x<Z_{v}(D)$ and $v=0$ if $x>Z_{u}(D)$. Furthermore, since $L>A$ and $V$ is strictly convex, $Z_{u}(D)>Z_{v}(D)$.

\section{Behavior of the policy when the demand is low}

Now, if the demand is low, when $x>Z_{u}(L), u=0$ and $v=0$. Similarly, when $Z_{u}(L)>x>Z_{v}(L), u=\mu$ and $v=0$. Finally, when $x<Z_{v}(L), u=\mu$ and $v=\mu_{c}$. When $x=Z_{u}(L)$ and $D=L$, since $\mu>\mu_{L}$ and $v=0$, equation (4) shows that $d x / d t>0$. Therefore, $x$ increases and soon exceeds $Z_{u}(L)$. However, when $x>Z_{u}(L)$, $u$ is set to zero and $x$ decreases to $Z_{u}(L)$. Consequently, $u$ will jump infinitely rapidly between $o$ and $\mu$ and $\mathrm{x}$ will remain very close to $\mathrm{Z}_{\mathrm{u}}(\mathrm{L})$. To avoid this unnecessary chattering, we choose $u=\mu$ when $x=Z_{u}(L)$. With this choice, $d x / d t=0$ and $x$ remains at $Z_{u}(L)$ until the demand changes to high. Therefore $Z_{u}(L)$ is a hedging point. Since there is a backlog cost when $x<0$, setting $\mathrm{Z}_{\mathbf{u}}(\mathrm{L})<0$ will incur a backlog cost with no offsetting benefits. Consequently, we can assume that $\mathrm{Z}_{\mathbf{u}}(\mathrm{L}) \geq \mathbf{0}$.

\section{Behavior of the policy when the demand is high}

Similarly, when $x=Z_{u}(H)$ and $D=H$, since $\mu<\mu_{H}$ and $v=0$, equation (4) shows that $d x / d t<0$. This implies that when the demand is high, inventory/surplus decreases and it cannot stay at $x=Z_{u}(H)$ and $Z_{u}(H)$ is not a hedging point. As $x$ decreases, it eventually reaches $Z_{v}(H)$. When $\mathbf{x}<\mathbf{Z}_{\mathbf{v}}(\mathbf{H})$, since $\mu+\mu_{c} \geq \mu_{\mathbf{H}}$, equation (4) shows that $\mathbf{d x} / \mathbf{d t}>0$. However, as $\mathbf{x}$ increases, it will soon exceed $Z_{v}(H)$ and $v$ is set to $o$. Similar to the behavior of $u$ at $x=Z_{u}(L), v$ will jump infinitely rapidly between $o$ and $\mu_{c}$ and $\mathrm{x}$ will remain very close to $\mathrm{Z}_{\mathrm{v}}(\mathrm{H})$. To avoid this unnecessary chattering, we choose $v=\mu_{H^{-}} \mu$ when $x=Z_{v}(H)$. With this choice, $d x / d t=0$ and $x$ remains at $Z_{v}(H)$ until the demand changes to low. As a result $\mathrm{Z}_{\mathrm{v}}(\mathrm{H})$ is the other hedging point. Since there is an inventory carrying cost when $x>0$, setting $Z_{v}(H)>0$ will incur an inventory carrying cost with no offsetting benefits. Therefore, we can assume that $\mathrm{Z}_{\mathrm{v}}(\mathrm{H}) \leq \mathrm{o}$.

Note that when $x>Z_{u}(L)$, $x$ decreases regardless of the demand state. Similarly, when $x<Z_{v}(H)$, it increases for both demand states. Consequently, the regions $x>Z_{u}(L)$ and $x<Z_{v}(H)$ are 
transient and $x$ is bounded between the upper and lower hedging levels, i.e., $Z_{v}(H) \leq x \leq Z_{v}(H)$ in the steady state.

\section{Summary of the Optimal Policy}

Finally, we can summarize the optimal policy. Let us rename $Z_{u}(L)$ as $Z$ and $Z_{v}(H)$ as $S$ for simplicity. The optimal policy is a $(\mathrm{Z}, \mathrm{S})$ policy. The manufacturer produces with the maximum production rate until the finished goods inventory reaches $\mathrm{Z}$. At this level, the manufacturing facility produces at the demand rate to keep $\mathrm{x}$ at this level. The subcontractor is used only when the backlog level reaches $\mathrm{S}$. At this level, the subcontractor is requested to supply goods at a rate of $\mu_{\mathrm{H}}-\mu$. This policy keeps $\mathrm{x}(\mathrm{t})$ bounded between $\mathrm{Z}$ and $\mathrm{S}$.

Given the optimal policy, the steady state behavior of the system when it is controlled by this policy is analyzed in the next section. Once the performance is evaluated for given values of $Z$ and $S$, the objective function will be evaluated and maximized to determine the optimal values of $\mathrm{Z}$ and $\mathrm{S}$.

\section{Analysis of the Model}

Figure I shows sample realizations for the cases where only the manufacturing facility is used and both the manufacturing facility and the subcontractor are used. Figure 4 depicts the cumulative production and demand for these cases.

The performance analysis of the model is carried by determining the differential equations that explain the behavior of the system in the interior states and solving these equations subject to some boundary conditions.

\section{Probability Distribution}

When the amount of finished goods inventory is not equal to the hedging level and it is not zero, the system is said to be in the interior region. In this region, the system state at time $t, S(t)$, is expressed by a tuple $(D(t), X(t))$ where $D(t) \in\{H, L\}$ and $S \leq x(t) \leq Z$.

The time dependent system state probabilities for the interior region, $F_{i}(t, x)$ is defined as

$$
\left.\mathrm{F}_{\mathbf{i}}(\mathrm{t}, \mathbf{x})=\mathrm{P}[\mathrm{D}(\mathrm{t})=\mathbf{i}, \mathbf{x}(\mathrm{t}) \leq \mathbf{x}\}\right] \quad \mathrm{i} \in\{\mathrm{H}, \mathrm{L}\}, \mathrm{t} \geq \mathbf{0}, \mathrm{S}<\boldsymbol{x}<\mathrm{Z} .
$$

The time-dependent system state density functions are also defined as:

$$
f_{i}(t, x)=\frac{\partial F_{i}(t, x)}{\partial x} \quad \mathbf{i} \in\{\mathbf{H}, \mathbf{L}\}, \mathbf{t} \geq \mathbf{o}, \mathbf{S}<\boldsymbol{x}<\mathbf{Z} .
$$



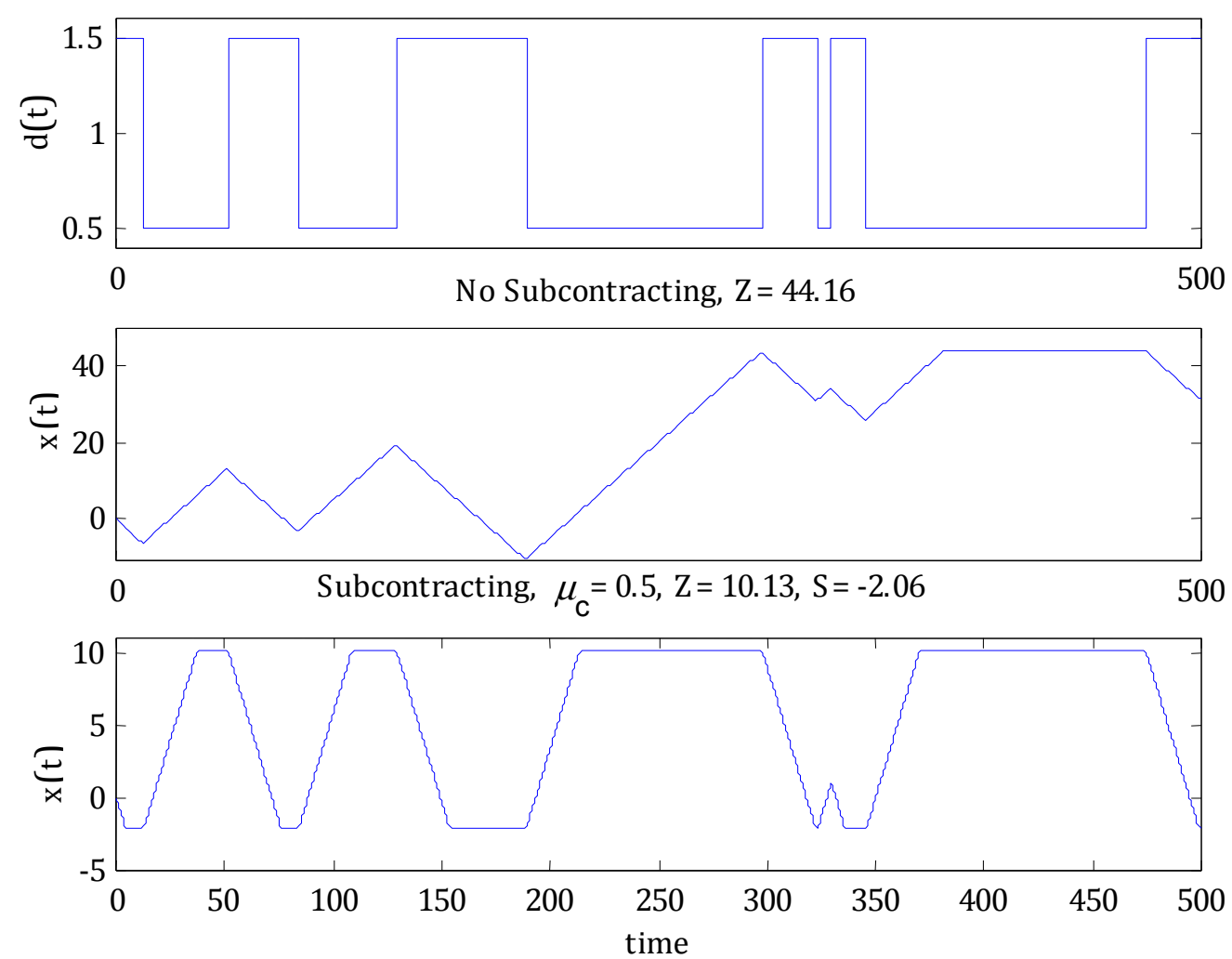

Figure 3. The effect of the option on the surplus/backlog. $\mu=\mathbf{I}, \mu_{\mathrm{H}}=\mathbf{I} .5, \mu_{\mathrm{L}}=\mathbf{0 . 5}, \bar{d}=0.9, \quad \mathbf{c v}=$ $3, c^{-}=0.08, c^{+}=0.24, \mathrm{~L}-\mathrm{A}=9$.

No Subcontracting, $Z=44.16$






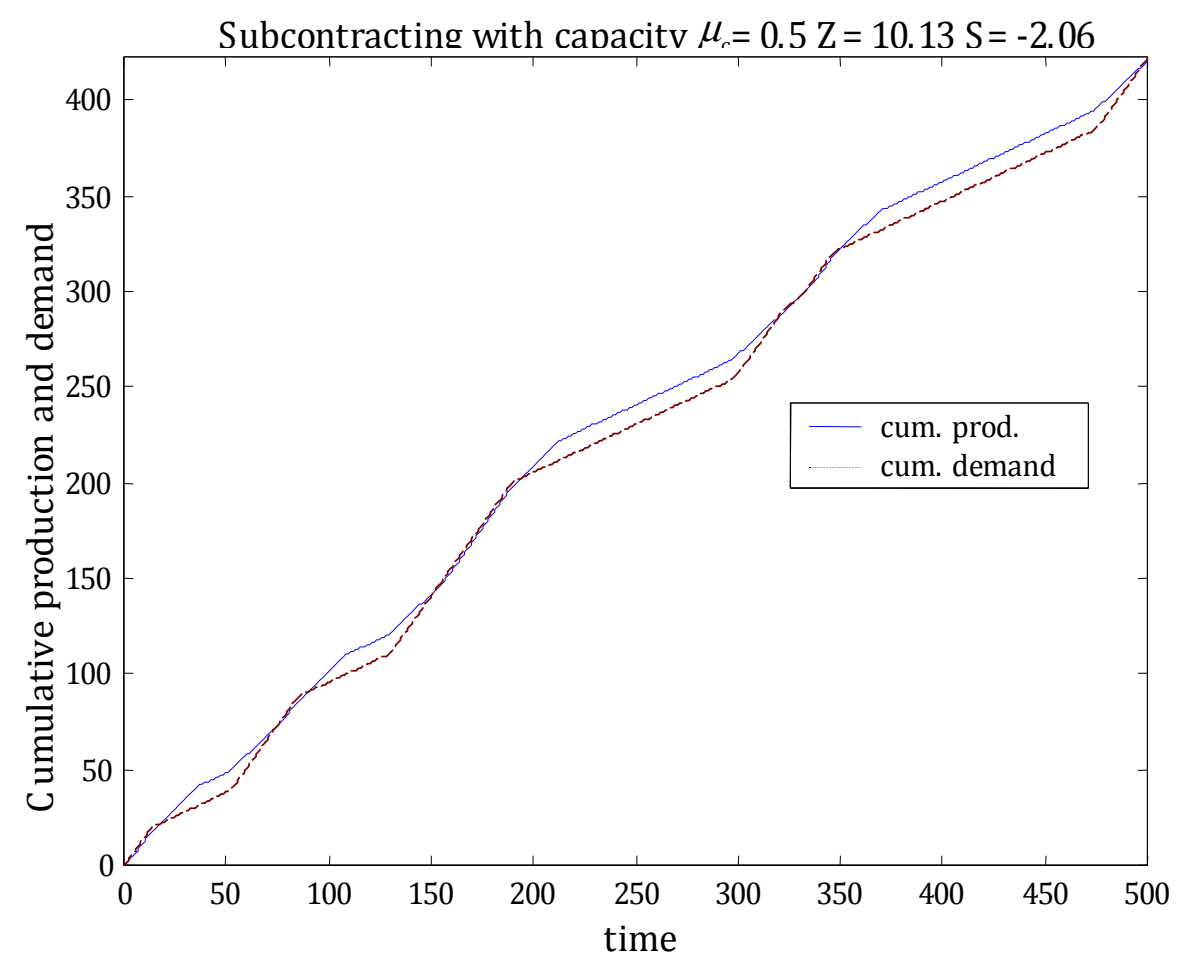

Figure 4. The effect of the option on the cumulative demand and production. $\mu=\mathbf{I}, \mu_{\mathrm{H}}=\mathbf{I} .5, \quad \mu_{\mathrm{L}}=$ $0.5, \bar{d}=0.9, \mathrm{cv}=3, \mathrm{c}^{-}=\mathbf{0 . 0 8}, \mathrm{c}^{+}=\mathbf{0 . 2 4}, \mathrm{L}-\mathrm{A}=\mathbf{9}$.

We assume that the process is ergodic and, thus, the steady-state density functions exist. The steady-state density functions are defined as:

$$
f_{i}(x)=\lim _{t \rightarrow \infty} f_{i}(t, x) \quad \mathbf{i} \in\{\mathbf{H}, \mathbf{L}\}, \mathbf{t} \geq \mathbf{o}, \mathbf{S}<\boldsymbol{x}<\mathbf{Z} .
$$

The probabilities that the finished goods inventory is equal to the produce-up-to level and $S, P_{Z}$ and $P_{S}$ are given as

$$
\begin{aligned}
& P_{Z}=\lim _{t \rightarrow \infty} P[x(t)=Z] \\
& P_{S}=\lim _{t \rightarrow \infty} P[x(t)=S]
\end{aligned}
$$

We first consider the behavior of the process in the interior regions $S<\mathbf{X}<\mathrm{Z}$. Conditioning the probability density of the event $\{(D(t+\delta t), X(t+\delta t))=(H, x)\}$ on the state of the system at time $t$ yields

$$
f_{\mathbf{H}}(t+\delta t, x)=f_{\mathbf{H}}\left(t, x-\left(\mu-\mu_{\mathbf{H}}\right) \delta t\right)(1-p \delta t)+f_{\mathbf{L}}\left(t, x-\left(\mu-\mu_{\mathbf{L}}\right) \delta t\right)(r \delta t)+o(\delta t) \quad(\mathbf{I} 7)
$$


where $o(\delta t)$ approaches to zero faster than $\delta$. The above equation can be written in differential form for $\delta t \rightarrow o$ as

$$
\frac{\partial f_{\mathbf{H}}(t, x)}{\partial t}+\left(\mu-\mu_{\mathbf{H}}\right) \frac{\partial f_{\mathbf{U}}(t, x)}{\partial x}=-p f_{\mathbf{H}}(t, x)+r f_{\mathbf{L}}(t, x)
$$

Taking the limit of the above equation as $t \rightarrow \infty$ yields the following differential equation for $f_{H}$

$$
\left(\mu-\mu_{\mathbf{H}}\right) \frac{d f_{\mathbf{H}}(x)}{d x}=-p f_{\mathbf{H}}(x)+r f_{\mathbf{L}}(x)
$$

Following the same steps for $f_{L}$ yields

$$
\left(\mu-\mu_{\mathbf{L}}\right) \frac{d f_{\mathbf{L}}(x)}{d x}=p f_{\mathbf{H}}(x)-r f_{\mathbf{L}}(x)
$$

Note that, since $\mu_{\mathrm{L}}<\mu<\mu_{\mathrm{H}}$, the inventory level decreases when the demand is high and increases when demand is low.

In order to solve the set of first order differential equations given in (19) and (20), two boundary conditions are needed. First, note that at any given level of the finished goods inventory, the number of upward crossings must be equal to the number of downward crossings. Let $\quad \mathrm{N}(\mathrm{i}, \xi, \mathrm{T})$ denote the total number of level crossings in state $i$, at surplus level $\xi$, in the time interval $[t, t+T]$ for large $T$. Then

$$
\lim _{T \rightarrow \infty} N(\mathbf{H}, \xi, T)=\lim _{T \rightarrow \infty} N(L, \xi, T)
$$

The renewal analysis shows that

$$
\lim _{T \rightarrow \infty} \frac{N(i, \xi, T)}{T}=\Delta_{r} f_{i}(\xi)
$$

where $\Delta_{r}$ is the rate of change in the buffer level at state $i$, and $f_{i}(x)$ is the steady-state density function $^{25}$. Then, equation (2I) can be written as

$$
\left(\mu_{\mathbf{H}}-\mu\right) f_{\mathbf{H}}(x)=\left(\mu-\mu_{\mathbf{L}}\right) f_{\mathbf{L}}(x) \text {. }
$$

Using this result in equation (19) gives the following first order differential equation

$$
\frac{d f_{\mathbf{H}}(x)}{d x}=\left(\frac{p}{\mu_{\mathbf{H}}-\mu}-\frac{r}{\mu-\mu_{\mathbf{L}}}\right) f_{\mathbf{H}}(x)
$$

whose solution is

$$
f_{\mathbf{H}}(x)=c \cdot e^{\lambda x}
$$


where $\lambda=\frac{p}{\mu_{\mathbf{H}}-\mu}-\frac{r}{\mu-\mu_{\mathrm{L}}}$ and $\mathrm{c}$ is a constant to be determined. Following equation (23),

$$
f_{\mathbf{L}}(x)=c \frac{\mu_{\mathbf{H}}-\mu}{\mu-\mu_{\mathbf{L}}} e^{\lambda x}
$$

Now consider the probability that the finished goods inventory is equal to the produce-upto level; $\mathbf{P}_{\mathrm{Z}}$. Since $\mu_{\mathrm{L}}<\mu<\mu_{\mathrm{H}}$, the inventory level can reach this level only when the demand is low, so the manufacturing capacity can add to the finished goods buffer. Each time the inventory level increases and reaches the level $\mathrm{Z}$, it stays there for a period until the state of the demand changes to high and the inventory level starts decreasing. This time is equal to $\mathrm{I} / \mathrm{r}$. By the ergodicity argument, $P_{Z}$ is equal to the time average of the total sojourn in this state:

$$
P_{Z}=\lim _{T \rightarrow \infty} \frac{N(\mathbf{L}, Z, T)}{T} \frac{1}{r}=\left(\mu-\mu_{\mathbf{L}}\right) f_{\mathbf{L}}(Z) \frac{1}{r}=c \frac{\mu_{\mathbf{H}}-\mu}{r} e^{\lambda x}
$$

Similarly

$$
P_{S}=\lim _{T \rightarrow \infty} \frac{N(\mathbf{H}, S, T)}{T} \frac{1}{p}=\left(\mu_{\mathbf{H}}-\mu\right) f_{\mathbf{H}}(S) \frac{1}{p}=c \frac{\mu_{\mathbf{H}}-\mu}{p} e^{\lambda S}
$$

Finally, the constant $\mathrm{c}$ is determined by using the normalizing condition. Namely, the sum of all the probabilities must add up to I:

$$
\int_{S}^{Z}\left[f_{\mathbf{H}}(x)+f_{\mathbf{L}}(x)\right] d x+P_{Z}+P_{S}=1
$$

Using equations (25), (26), (27), and (28) with the normalizing condition given above yields

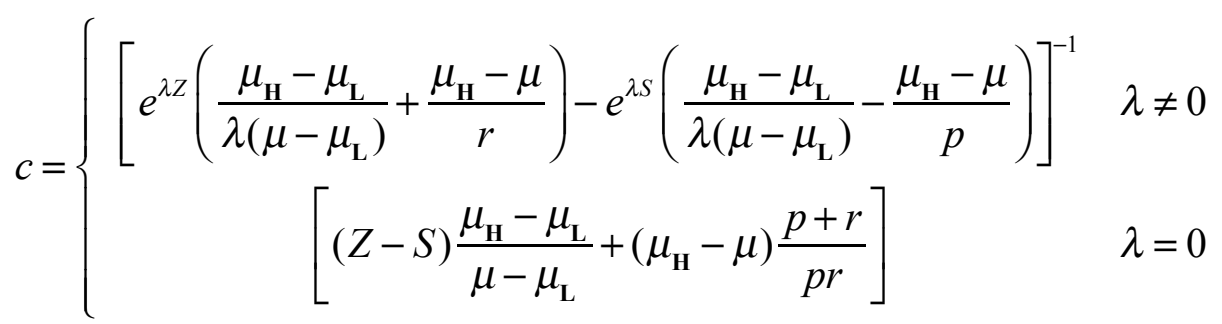

\section{Evaluation of the objective function and other performance measures}

Now, since the complete solution of the system is available in the derived density functions and the probability masses, the performance measures of interest can be determined. One of the most important performance measures is the total sales per time TH. Since backlog is allowed, TH is equal to the average demand rate.

The average finished goods inventory WIP is 


$$
\mathbf{W I P}=\int_{0}^{Z}\left[x f_{\mathbf{H}}(x)+x f_{\mathbf{L}}(x)\right] d x+Z \cdot P_{Z}
$$

which is evaluated as

$$
\mathbf{W I P}=\left\{\begin{array}{cc}
c\left[\mathbf{Z e}^{\left.\lambda \mathbf{Z}\left(\frac{\mu_{\mathbf{H}}-\mu_{\mathbf{L}}}{\lambda\left(\mu-\mu_{\mathbf{L}}\right)}+\frac{\mu_{\mathbf{H}}-\mu}{r}\right)-\mathbf{e}^{\lambda \mathbf{Z}} \frac{\mu_{\mathbf{H}}-\mu_{\mathbf{L}}}{\lambda^{2}\left(\mu-\mu_{\mathbf{L}}\right)}+\frac{\mu_{\mathbf{H}}-\mu_{\mathbf{L}}}{\lambda^{2}\left(\mu-\mu_{\mathbf{L}}\right)}\right]}\right. & \lambda \neq 0 \\
c \cdot\left[\mathbf{Z}^{2} \frac{\mu_{\mathbf{H}}-\mu_{\mathbf{L}}}{2\left(\mu-\mu_{\mathbf{L}}\right)}+Z \frac{\mu_{\mathbf{H}}-\mu}{r}\right] & \lambda=0
\end{array}\right.
$$

Similarly, the average backlog level BG is

$$
\mathbf{B G}=E\left[x^{-}\right]=-\int_{S}^{0}\left[x f_{\mathbf{H}}(x)+x f_{\mathbf{L}}(x)\right] d x-S \cdot P_{S}
$$

which is evaluated as

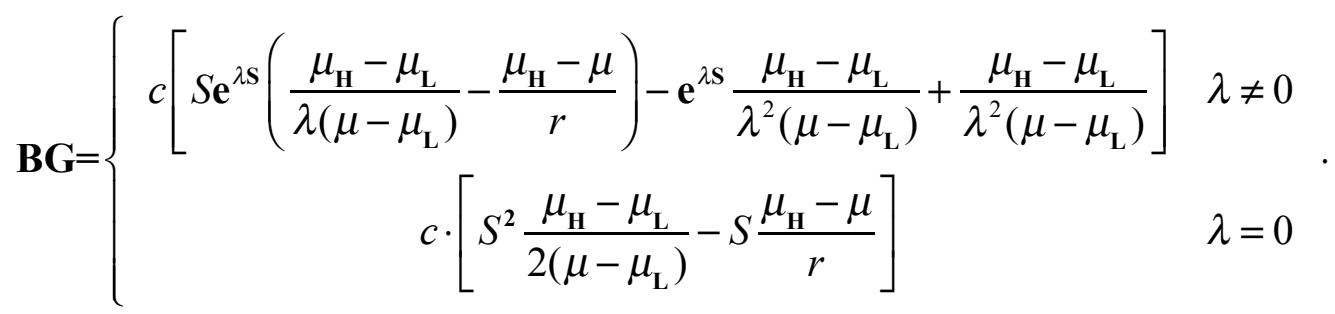

The average rate at which the subcontractor supplies goods is

$$
\mathbf{T H}_{v}=\left(\mu_{\mathbf{H}}-\mu\right) P_{S}=c\left(\mu_{\mathbf{H}}-\mu\right)\left(\mu-\mu_{\mathbf{L}}\right) e^{\lambda S}
$$

Finally the optimal profit is

$$
\Pi_{1}^{*}=L \cdot \mathbf{T H}-(L-A) \cdot \mathbf{T H}{ }_{v}-c^{+} \mathbf{W I P}-c^{-} \mathbf{B G}
$$

\section{Evaluating the Terms of a Given Contract}

A capacity option is specified by its option price $C_{O}$, the exercise price $\Delta_{c}$, and its duration $\Gamma$. In the derivation of the optimal policy, we assume that $(x, D)$ reaches steady state. Consequently, let us assume that the duration of the option is long enough to reach the steady state.

In the preceding analysis, the exercise price of the option is taken into account to determine $\Pi_{\mathrm{I}}^{*}$. Under the steady state assumption, since $\Pi_{\mathrm{I}}^{\prime \prime}$ is the profit per unit time, the net profit obtained by using an option in the duration of the agreement is $\Pi_{1}^{*} \Gamma-C_{0}$.

Let $\Pi_{2}^{*}$ be the profit obtained if only the manufacturing facility is used without the subcontractor. The contractual agreement with the subcontractor is beneficial only if $\Pi_{1}^{*} \Gamma-\mathrm{C}_{\mathrm{O}}>\Pi^{*}{ }_{2} \Gamma$, or equivalently if 


$$
\mathrm{C}_{\mathrm{O}}<\left(\Pi^{*}{ }_{\mathrm{I}}-\Pi_{2}^{*}\right) T
$$

The above inequality can be utilized to evaluate the terms of a given contractual agreement.

Figure 5 shows how the terms of the contract can be evaluated by using the relationship between the additional cost, gain in the profit, and the fixed cost and the duration of the option. For example, the figure shows that, it is possible to increase the profits by paying $20 \%$ of the expected profit without using the subcontractor during the duration of the option as an upfront payment (option price) and paying less than $120 \%$ of the regular production cost as you receive goods from the subcontractor (exercise price of the option).

Figure 6 illustrates the effects of demand variability on a specific option with an upfront payment of $20 \%$ of the profit without subcontracting and an exercise price which is $50 \%$ above the production cost. As the demand variability, summarized with its coefficient of variation, increases the value of the option increases. However, for low variability cases $\mathrm{cv}<0.9$, it is not profitable to use the option since the company cannot justify the upfront payment for these cases.

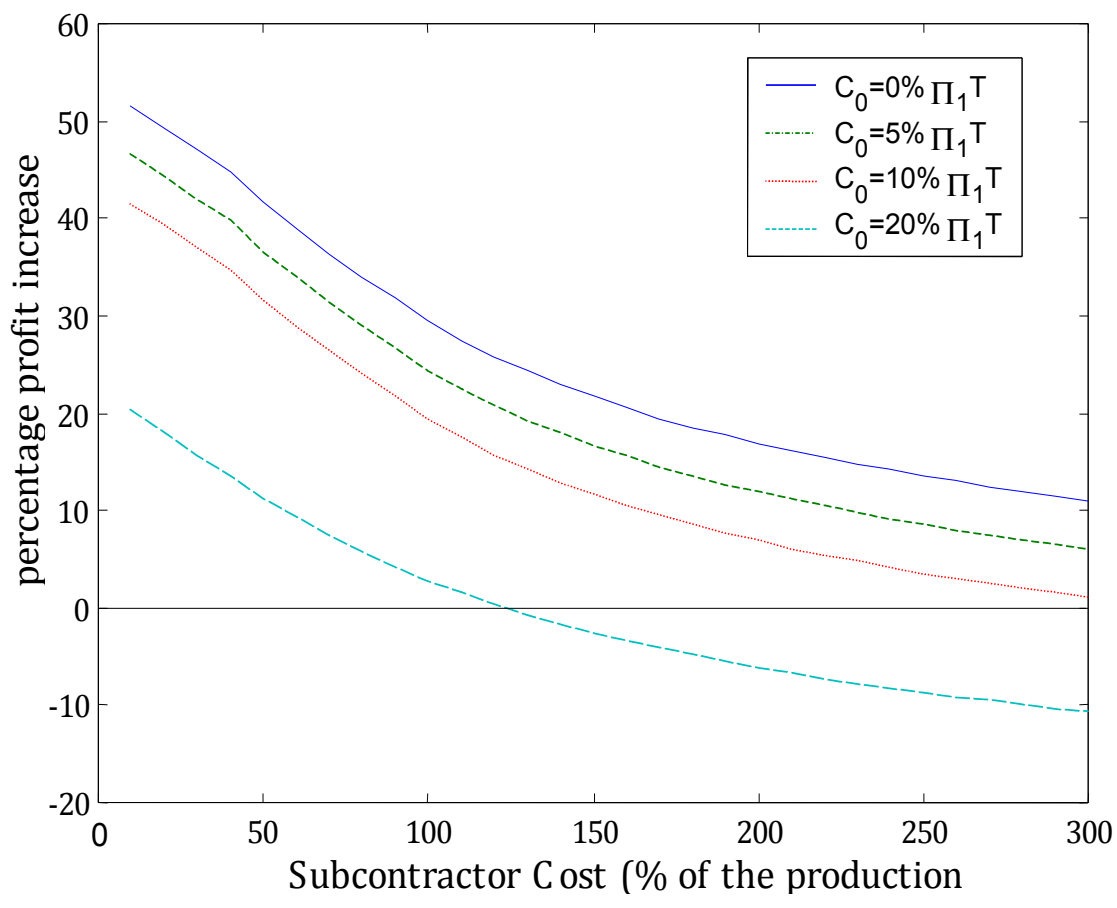

Figure 5. Evaluating the terms of the contract: fixed payment and additional production cost

$$
\left(\mu=\mathbf{I}, \mu_{\mathrm{H}}=\mathbf{I} .5, \mu_{\mathrm{L}}=\mathbf{0 . 8}, \bar{d}=0.9, \mathbf{c v}=\mathbf{I}, \mathbf{c}^{-}=\mathbf{0 . 3}, c^{+}=\mathbf{0 . I}, \mathbf{L}=3, \mathbf{c}_{\mathrm{p}}=6\right)
$$




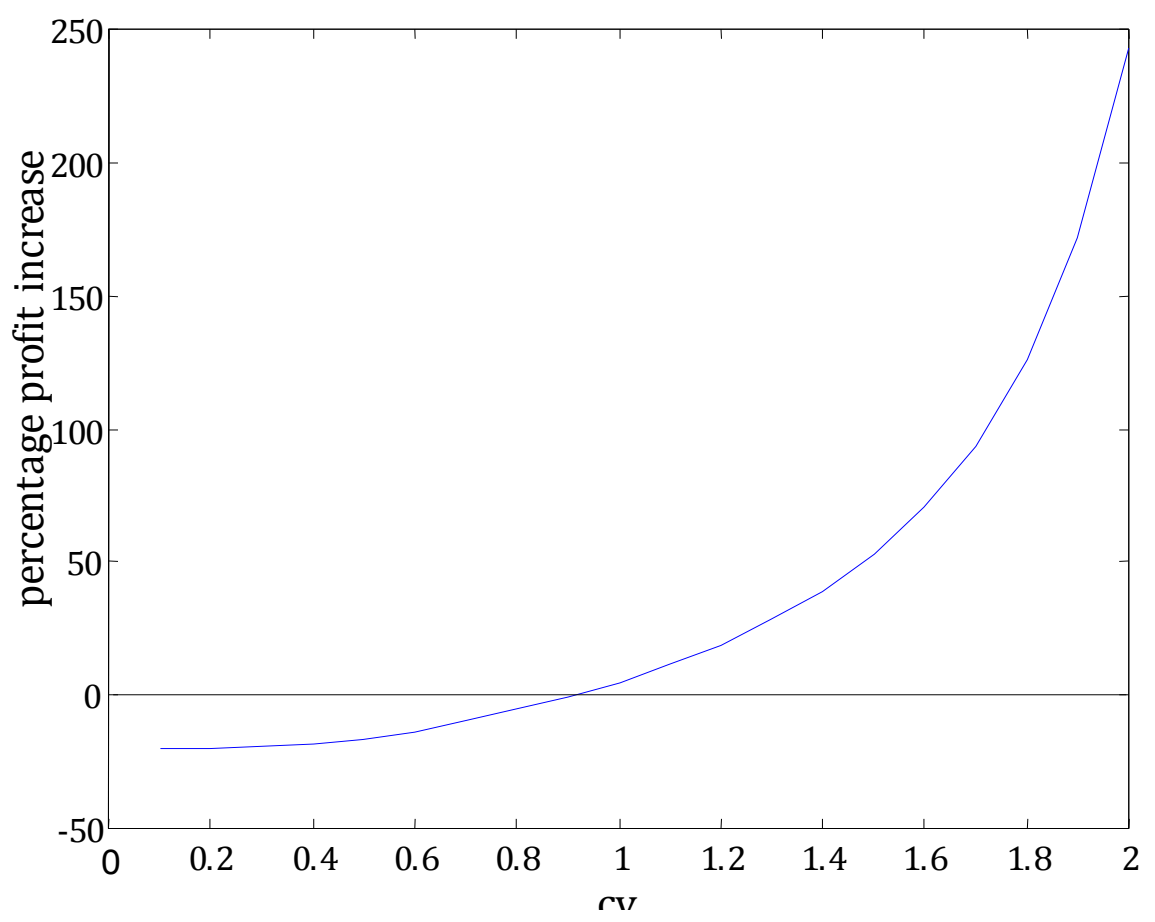

Figure 6. Effects of demand variability on the Additional Profit $\left(\mu=\mathbf{I}, \mu_{\mathrm{H}}=\mathbf{I . 5}, \mu_{\mathrm{L}}=\mathbf{0 . 8}, \bar{d}=0.9\right.$, $\left.\mathrm{cv}=\mathrm{I}, \mathrm{c}^{-}=0.3, c^{+}=0 . \mathrm{I}, \mathrm{L}=5, \mathrm{~A}=2, \mathrm{C}_{0}=20 \% \Pi_{\mathrm{I}} \mathrm{T}\right)$

\section{Concluding Remarks}

In this study, we investigated the strategy of using capacity options to manage the demand volatility risks for manufacturers that produce a replenishment product that is sold at a retailer. The model shows analytically that option-type contractual agreements can be used as a strategy to cope with demand variability. Furthermore, it is observed that the value of this strategy increases as the demand uncertainty increases. This result supports the options view that uncertainty creates opportunity. Furthermore the additional costs of sourcing from a short-cycle manufacturer can be justified through increased sales and reduced finished goods inventories. All of these conclusions are based on a detailed analytical model that captures the dynamics of the system controlled by the optimal production and subcontracting policies. The same framework can also be used to value different production resources with different capacities and operating costs in the same factory. This allows one to value volume flexibility.

Furthermore, the model can be extended in a number of ways. First, the model considered in this study requires that the demand rate can be met by production, and thus the model is feasible. Relaxing this assumption requires modeling the customer behavior directly. More specifically, if the customer demand is sensitive to the backlog level and some of the customers are lost if the quoted waiting time is excessive, the system reaches an equilibrium where the capacity is matched with the demand of the customers who decide to order. This extension also makes it unnecessary to include the backorder cost which is not a tangible cost in the objective function. 
The effect of customer service is reflected in the objective function through lost sales and thus lost profits. This extension is currently under investigation.

Secondly, the model can be extended to consider multiple subcontractors. It is also desirable to include delays into the model. However, including delays in the stochastic control problem directly is quite challenging.

The strategy of temporarily increasing the production capacity is more advantageous for the producer. Subcontractors should have enough incentives to take part in such agreements. The upfront payment of the option may provide such an incentive. In order to analyze the manufacturer-subcontractor relations more thoroughly, the framework needs to be extended to include competition among subcontractors and also among producers. Furthermore, the approach can be extended to investigate capacity expansion decisions of the producers and the investment decisions in short-cycle manufacturing. These extensions are left for future research.

If the assumptions of the model cannot be justified to use the results immediately in an application, a more detailed simulation model that uses the optimal production and subcontracting policy derived in this study and a simulation-based optimization method can be used to value the capacity option.

\section{Acknowledgements}

The author acknowledges the support of the TUBITAK-NATO Science Fellowship program. The author thanks the members of the Harvard Center for Textile and Apparel Research for the many interesting and helpful discussions and S.B.Gershwin for his valuable comments.

\section{References}

I. Abernathy FH, Dunlop JT, Hammond JH, and Weil D (1999). A Stitch in Time Oxford University Press, New York: New York.

2. Fisher M., Hammond JH, Obermeyer WR, and Raman A (1994). Making supply meet demand in an uncertain world. Harv Bus Rev May-June: 83-93.

3. Hunter N, King RE, and Nuttle HLW (1996). Evaluation of traditional and quick response retailing procedures using stochastic simulation model. J Text Inst 87: 42-55.

4. Abernathy FH, Dunlop JT, Hammond JH, and Weil D (2000). Control your inventory in a world of lean retailing. Harv Bus Rev November-December: I69-I76.

5. Amram M and Kulatilaka N (1999). Real Options: Managing Strategic Investment in an Uncertain World Harvard Business School Press: Boston, MA

6. Bielecki $T$ and Kumar PR (1988). Optimality if zero-inventory policies for unreliable manufacturing systems. Opns Res 36:532-54I.

7. Huang L, Hu JQ and Vakili P (1999). Optimal control of a multi-state manufacturing system: control of production rate and temporary increase in capacity. In: Proceedings of the $2^{\text {nd }}$ Aegean International Conference on Analysis and Modeling of Manufacturing Systems: Greece, pp I9I-198. 
8. Jain K and Silver EA (1995). The single-period procurement problem where dedicated supplier capacity can be reserved. Nav Res Log 42: 915-934.

9. Costa D and Silver EA (1996). Exact and approximate algorithms for the multi-period procurement problem where dedicated supplier capacity can be reserved. Opns Res Spec 18:197207.

Io. Eppen GD and Iyer AV (1997). Backup agreements in fashion buying-the value of upstream flexibility. Mgmt Sci 43: I469-I484.

II. Bassok $Y$ and Anupindi R (1997). Analysis of supply contracts with total minimum commitments. IIE Trans 29:373-38I.

I2. Anupindi R and Bassok Y (1998). Supply contracts with quantity commitments and stochastic demand. In: Tayur S., Magazine M, and Ganeshan R (eds). Quantitative Models in Supply Chain Management: Kluwer Academic Publisher.

I3. Tsay AA and Lovejoy W (1999). The quantity flexibility contract and supply chain performance. Mnfg Ser Oper Mngmt I: 89-III.

14. Brown AO and Lee HL (1997). Optimal pay-to-delay capacity reservation with application to the semiconductor industry. Working Paper, Department of Industrial Engineering and Engineering Management, Stanford University, Stanford, CA.

I5. Barnes-Schuster DC, Bassok Y, and Anupindi R (200o). Coordination and flexibility in supply contracts with options. unpublished working paper.

I6. Gershwin SB (1994a). Extension of FMS scheduling model. unpublished note.

17. Whittemore AS and Saunders SC (1977). Optimal inventory under stochastic demand with two supply options. SIAM J App Math 32: 293-305.

18. Zhang V (1995). Ordering policies for an inventory system with supply flexibility. PhD thesis, Stanford University, Department of Industrial Engineering and Engineering Management.

19. Krichagina EV, Lou SXC and Taksar MI (1994). Double band policy for stochastic manufacturing systems in heavy traffic. Math of OR 19:560-597.

20. Bradley JR (1999). Optimal control of an M/M/I subcontracting model. Technical Report, Cornell University.

2I. Bradley JR and Glynn PW (2000). Managing the Manufacturer-Subcontractor Relationship and the Manufacturer's Optimal Capacity, Inventory, and Subcontracting Policies. Technical Report, Cornell University.

22. Van Mieghem JA (1999). Coordinating investment, production, and subcontracting. Mgmt Sci 45: 954-971.

23. Tan B (1997). Variance of the throughput of an $\mathrm{N}$-station production line with no intermediate buffers and time dependent failures. Eur J Opl Res I0I:.560-576.

24. Gershwin SB (1994b). Manufacturing Systems Engineering: Prentice Hall: Englewood Cliffs, NJ 07632.

25. Yeralan S and Tan B (1997). A station model for continuous materials flow production. Inter J Prod Res 35:2525-254I. 